\title{
Using System Dynamics to study Army Reserve deployment sustainability
}

\author{
Jun Wang \\ LCA, JOAD, DST Group \\ Email: jun.wang@dst.defence.gov.au
}

\begin{abstract}
The reserve forces have been playing increasingly important roles in the Defence capability of developed countries, due to the skill shortages in regular forces and defence budget pressures. The Australian Defence Force (ADF) in particular, is increasing the employment of its Reservists to deliver defence capability, including the deployed capability on operations within Australia and overseas. The Australian Army Reserve (ARes) is an important component of Force Generation in Plan BEERSHEBA, which is a government endorsed force-structure modernisation program.
\end{abstract}

This work presents a case study on the "raise, train and sustain" aspects of a deployed ARes unit using System Dynamics (SD) simulation. More specifically, the SD model constructed examines the sustainability of the deployment of a volunteer-based Army Reserve unit in a low-intensity regional stabilisation operation. The soldiers' behavioural change over time, in terms of willingness to deploy (represented as the volunteer percentage), is modelled and its impact on the volunteer availability for a continuous deployment is simulated.

With the subject matter experts (SMEs) input about soldiers' willingness to deploy based on their deployment experience, the SD model provides insights on the following questions:

- How many volunteers at the rank of Private (PTE) can a given pool of soldiers supply to support a deployed ARes unit?

- What is the required size of the pool of PTE soldiers to support a battalion-sized ARes unit in deployment?

The SD supply model showed that a pool with 1970 PTE soldiers can provide a deployed unit with 240 volunteers in the long term, but there are only 158 PTE volunteers for the sixth rotation. A pool with 660 PTEs can supply a deployed force with 80 volunteers in the long term, but there are only 53 PTEs volunteering at the sixth rotation. A sensitivity analyses was also conducted by considering the worst and the best cases of the volunteer percentages. The SD demand model showed that to support a battalion-sized ARes unit, which demands 240 PTE Volunteers, a pool of 2230 PTEs is required.

In conclusion, this study demonstrates the applicability of SD simulation in sustainability analyses of ARes deployment. The model can be useful for Army Commanders in planning deployment of a volunteer-based ARes unit, and facilitating policy exploration to boost soldiers' willingness to deploy.

Keywords: $\quad$ System dynamics, sustainability, Army Reserve deployment 


\section{INTRODUCTION}

Military reserve forces have been playing increasingly important roles in defense capabilities worldwide because of the personnel and skill shortages in regular forces and Defence budget pressures. The Australian Defence Force (ADF) in particular, is increasing the employment of its Reservists to deliver defence capability, including the deployed capability on operations within Australia and overseas. In the Australian Army force structure Plan BEERSHEBA, endorsed in Defence White Paper 2013, the six Reserve Brigades are paired up to support three Combat Brigades in it's regular force through the entire 'Ready, Readying, and Reset' force generation cycle.

This article presents a sustainability study, using System Dynamics (SD) simulation, of the deployment of an Australian Army Reserve (ARes) unit for a low-intensity regional stabilisation operation. A stand-alone ARes unit was to be deployed for the first time in several decades, for which a large component of the volunteer positions are at the rank of Private (PTE). In preparing personnel for first time deployment, $60 \%$ of those qualified PTEs volunteered to deploy. The issue of concern is the sustainability of the unit for continuing deployment. The volunteer percentage for subsequent rotations from those who have been deployed is likely to drop due to family and/or employment issues. Two SD models are built to investigate the sustainability by modelling the soldiers' willingness to deploy, which is represented by the Volunteer Percentage (VP) provided by the Subject Matter Experts (SMEs). More specifically, the SD supply model answers the question: "How many volunteers can a given pool of PTE soldiers provide for a deployed ARes unit?", while the SD demand model answers the question: "What is the required size of the pool of PTE soldiers to support a given-size ARes unit in deployment?".

The article is organized as follows. Section 2 provides the background of SD simulation. Sections 3 and 4 present two SD models and the results. The conclusion is given in the last section.

\section{SYSTEM DYNAMICS SIMULATION}

SD, first started with the name "Industrial Dynamics" (Forrester, 1961), originated from the theory of nonlinear dynamics and feedback control of mathematics, physics and engineering (Sterman, 2000). It can be used to study "the information-feedback characteristics of industrial activity to show how organisational structure, amplification (in policies), and time delays (in decisions and actions) interact to influence the success of the enterprise (Forrester, 1961)".

Two diagramming tools used in SD modelling are Causal Loop Diagram (CLD) and Stock Flow Diagram (SFD). A CLD is used for qualitative analyses by examining the cause and effect relationships in feedback structures of a system, while a SFD is built to quantitatively simulate a system's dynamic behaviour (Sterman, 2000).

In a SFD, stock variables (also called state variables or levels) describe the states of the system, such as the number of soldiers, while flow variables (also called rate variables) depict the rates of change of stocks, such as the recruitment or separation rates. Stocks are accumulations of their flows and mathematically are calculated as the integration of net inflows.

\section{THE SD SUPPLY MODEL}

The SFD of the SD supply model is displayed in Figure 1. 


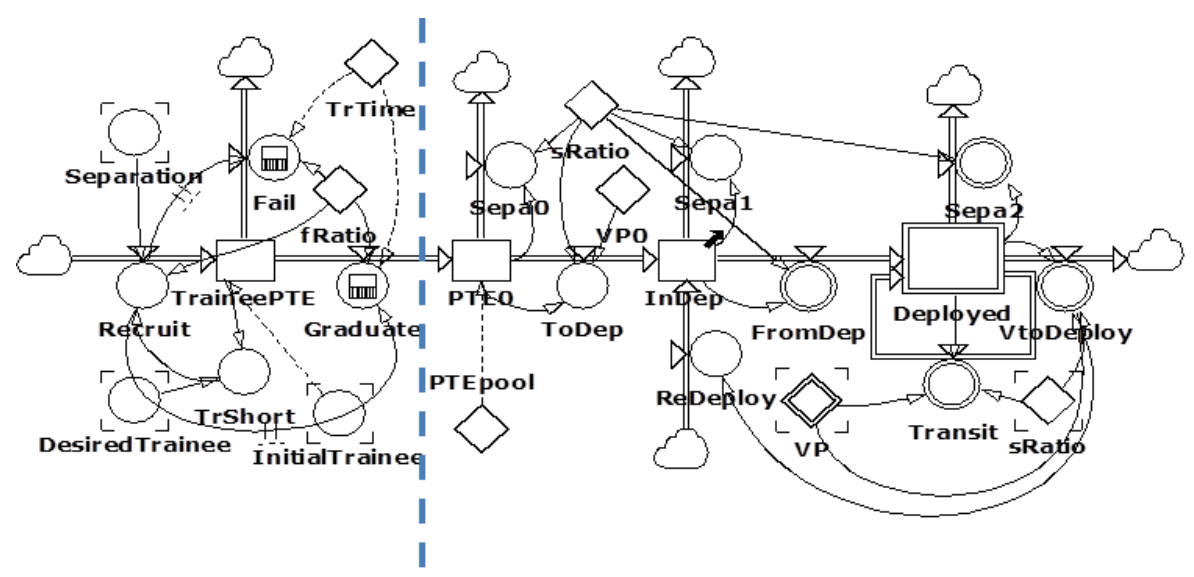

Figure 1. The SFD of the SD supply model

On the left side of the vertical dashed line in Figure 1, the trainees are recruited into the TraineePTE qualification course which takes two years (in part-time) to complete. With a $30 \%$ failure rate, $70 \%$ of the trainees become qualified. The function of this component is to model the training input, such that a stable pool of Privates is obtained.

On the right side of the vertical dashed line, PTEs are distributed in several states according to their deployment history, i.e., PTE0, InDep, and Deployed, which are all assumed to have a separation rate of $15 \%$ per year. PTE0 is the number of PTEs who have not been deployed. $V P_{0}$ percent of PTE0 soldiers join the soldiers in deployment (InDep). PTEs in InDep are deployed for a fixed-term, called a rotation (here assumed three months), and then rotated out to join the Deployed pool for those who have completed their deployment. The PTEs in the Deployed pool are further divided into 4 classes based on the time from their previous deployment. The PTEs in the class-i of the Deployed pool, Deployed $[i=1,2,3,4]$ are those who completed their last deployment less than or equal to one year, more than one but less than or equal to two years, more than two but less than or equal to three years, and more than three years. The PTEs in Deployed joins InDep with percentages $V P_{i}(\mathrm{i}=1,2,3,4)$ to redeploy. The model simulates the number of soldiers in deployment as the soldiers' behavioural change over time in terms of $V P_{i}$. The $V P_{i}$ used in Table 1 are from the SMEs' empirical input.

Table 1. The SME input for Volunteer Percentages.

\begin{tabular}{|c|c|c|c|c|c|}
\hline$i$ & 0 & 1 & 2 & 3 & 4 \\
\hline Years & 0 & $(0 \sim 1]$ & $(1 \sim 2]$ & $(2 \sim 3]$ & $(3+)$ \\
\hline$V P_{i}$ (Base) & $60 \%$ & $5 \%$ & $10 \%$ & $30 \%$ & $50 \%$ \\
\hline
\end{tabular}

The SD supply model can be used with different sizes of PTEpool to examine the number of volunteers a given PTEpool can provide. The simulation results are displayed in Figure 2. This provides an indication of the ability of the ARes unit to sustain a force element in continuing deployment.

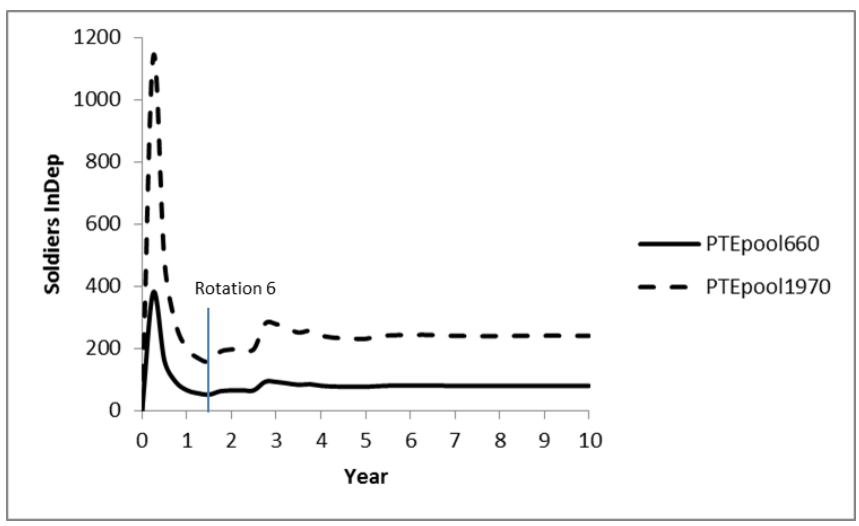

Figure 2. Number of volunteers for deployment. 
The simulation showed that a PTEpool with 1970 soldiers can provide a deployed force with 240 volunteer PTEs (about the required number of PTEs in a battalion) in the long term, but there are only 158 PTE volunteers for Rotation 6. A PTEpool with 660 soldiers can provide a company-sized ARes unit with 80 volunteer PTEs in the long term, but there are only 53 PTEs volunteering at Rotation 6.

For the sensitivity analysis, the SD supply model was rerun using $V P_{i}$ in Table 2, which were provided by the SMEs, for the best and worst cases. The results are displayed in Figure 3.

Table 2. Volunteer Percentages for the worst and best cases from the SMEs.

\begin{tabular}{|c|c|c|c|c|c|}
\hline$i$ & 0 & 1 & 2 & 3 & 4 \\
\hline years & 0 & $(0 \sim 1]$ & $(1 \sim 2]$ & $(2 \sim 3]$ & $(3+)$ \\
\hline$V P_{i}$ (Worst) & $30 \%$ & $2 \%$ & $10 \%$ & $15 \%$ & $20 \%$ \\
\hline$V P_{i}$ (Best) & $75 \%$ & $20 \%$ & $30 \%$ & $40 \%$ & $60 \%$ \\
\hline
\end{tabular}

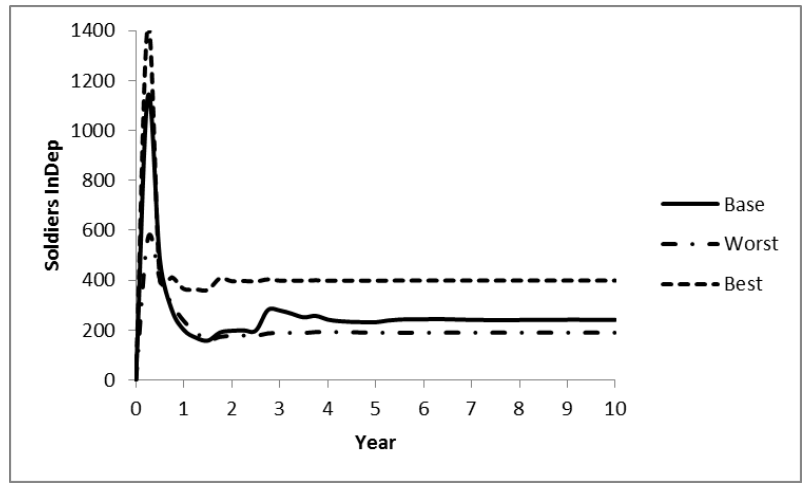

Figure 3. Volunteers supplied from a 1970 PTEpool with the Worst and Best $\boldsymbol{V} \boldsymbol{P}_{\boldsymbol{i}}$, and the Base case.

Figure 3 shows that a 1970 PTEpool, using the worst $V P_{i}$ in Table 2, can supply 190 volunteer PTEs for the deployed force in the long term, which is 50 PTEs less than that of the "Base case". This translates into approximately 2 concurrent company-sized deployments of PTEs. Using the best $V P_{i}$, the same PTEpool can supply 400 volunteer PTEs in the long run, which is 160 more than that of the "Base case". This translates into approximately 5 concurrent company-sized deployments of PTEs. The result raises questions about the ongoing sustainability of ARes operational deployments.

\section{THE SD DEMAND MODEL}

The SD demand model is an extension of the supply model in Figure 1. An extra stock variable Vpool is added to hold all volunteers waiting to be called to deploy in InDep. The stock InDep is a fixed size depending on the size of the deployed ARes unit. The following result is for a battalion-sized ARes unit deployment which requires 240 volunteer PTEs.

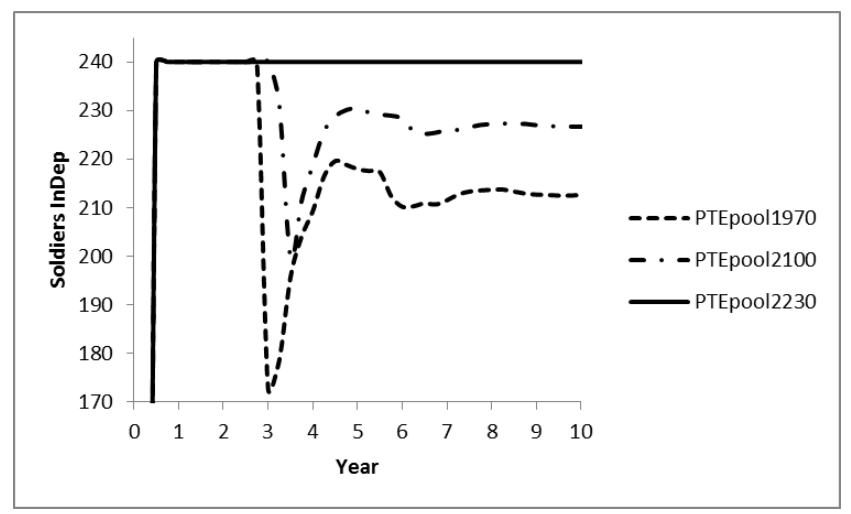

Figure 4. Volunteer PTEs provided by varying sizes of PTEpool 
Figure 4 suggests that a 1970 PTEpool can only support 10 rotations with the required 240 volunteer PTEs, but with a shortage of 27 PTEs in the long term. When the size of PTEpool increases to 2100, one more rotation can be supported but still with 14 PTEs less than that of the required. The PTEpool with 2230 PTEs is the minimum required size to provide 240 volunteers for the deployed battalion for the long-term continuous operation.

\section{CONCLUSION}

This article has presented two SD models used to analyse the sustainability of volunteer-based ARes deployment. The model simulation outputs can be used to inform the Army commanders about the sustainability issues regarding volunteer-based ARes deployment, and to promote policy exploration for improving volunteer percentages. Further work will investigate the application of system design techniques to improve the resilience of military workforce supply chains (MWSCs), where resilience is defined as "the ability of a system to return to its original state or to move to a new, more desirable state after being disturbed (Christopher and Peck, 2004)". A resilient MWSC is a fundamental component of military preparedness. "The Army Reserve is therefore critical for the future force generation of the nation's land forces. It will need to become a resilience and adaptive force, either reinforcing regular ground combat forces or providing a substantive part of the combat and general-purpose forces for subsequent force rotation (Smith and Palazzo, 2016)".

This study showed that SD models can provide a laboratory environment for the decision makers to test strategy options with aggregated stocks and flows. SD sees that system behaviour is determined by its structure, e.g., SFD, (Starr, 1980), which is essential to model the system of interest, in this case ARes deployment. The SD modelling learnt from this practice has already been used in the evaluation of Joint Fire Weapon systems (Wang et al., 2010). It is expected that the method could be applied in assessment of military autonomous systems in the future.

\section{ACKNOWLEDGMENTS}

The author would like to thank Dr. Richard Egudo and Ms. Jaci Pratt for their comments and advice in the preparation of this article. The author also would like to thank Dr. Aaron Jackson for his comments on the abstract.

\section{REFERENCES}

Christopher, M. \& Peck, H. (2004). Building the resilient supply chain. International Journal of Logistics Management, 15, 1-14.

Forrester, J. W. (1961). Industrial Dynamics, MIT Press.

Smith, C., Colonel \& Palazzo, A., Dr. (2016). Coming to terms with the modern way of war: Precision missiles and the land component of Australia's joint force. Australian Land Warfare Concept Series.

Starr, P. J. (1980). Modeling Issues and Decisions in System Dynamics. TIMS Studies in the Management Science, 14, 45-59.

Sterman, J. D. (2000). Business dynamics : systems thinking and modeling for a complex world, Boston, Mass., Irwin/McGraw-Hill.

Wang, J., Yue, Y., White, A., Major \& Egudo, R. (2010). Joint Fires - A System Dynamics Simulation Model for Assessing Joint Fires Force Packaging. In: PURI, V. \& FILIPPIDIS, D. (eds.) Land Warfare Conference 2010. Brisbane, Australia. 